\title{
Lack of allergic reactions in workers exposed to Pruteen (bacterial single-cell protein)
}

\author{
R W MAYES \\ From the Medical Department, Agricultural Division, Imperial Chemical Industries Ltd, \\ Billingham, Cleveland, UK
}

\begin{abstract}
Men exposed to high concentrations of bacterial single-cell protein (Pruteen) dust have complained of "sticky eyes" and influenza-like symptoms. Over four years, surveillance of a work force of 70 men with a programme that included a respiratory health questionnaire, skin prick testing, serum testing for precipitating antibodies, lung function measurements, and chest radiography has shown no evidence of allergic reaction or of exposure-related deterioration in lung function. The symptoms experienced may be attributable to exposure to lipopolysaccharide. The results show that Pruteen can be produced and handled without adverse effects on health, provided that adequate measures are taken to prevent exposure to high concentrations of dust.
\end{abstract}

Research and development on the production of single cell protein (Pruteen) has been carried out at Billingham during the past 12 years. In 1971 a pilot plant capable of producing 1000 tonnes a year was commissioned, and more than 100 employees have been concerned to some degree in producing and handling the product.

Pruteen is produced by the fermentation of methanol by Methylophilus methylotrophus, a Gram-negative aerobic organism that is dependent on methanol as its source of carbon. Extensive toxicological tests and animal feeding trials have shown it to be non-toxic and non-pathogenic. The fermentation is carried out in a pressure cycle fermenter as a monoculture in a substrate containing methanol, ammonia, and several trace elements. The process is continuous, the culture being drawn from the fermenter and the organism killed by acid and heat shock before being flocculated, dried, and oiled for dust suppression. The final product is in the form of granules for inclusion in the diet of pigs, poultry, and fish, or is ground to a fine powder for use as a milk substitute in the production of veal.

Pruteen in its final form is made up of dead bacterial cells in varying stages of distintegration and is composed of bacterial protein, nucleic acids, and lipopolysaccharide from the bacterial cell walls. The pilot plant is designed to operate with atmospheric dust concentrations below $1 \mathrm{mg} / \mathrm{m}^{3}$, and this is

Received 5 January 1981

Accepted 29 May 1981 monitored by repeated atmospheric testing as well as by personal monitoring at regular intervals. In areas where dust generation is unavoidable, such as during bagging, grinding, and maintenance work, the men are required to wear dust masks and goggles. The results of monitoring have shown that the hygiene level has been maintained during normal operation of the plant over the period of the survey.

\section{Clinical experience}

The main risk of exposure is from inhalation of the dust during production and handling. Experience of occupational exposure to biological dusts has shown that they may produce a variety of effects, including occupational asthma, extrinsic allergic alveolitis, byssinosis, and mill fever. ${ }^{1}$ More recently, exposure to proteolytic enzymes in the detergent industry has been shown to cause sensitisation. ${ }^{2}$ The introduction of any new biological dust in industry should be treated with caution because of the difficulty in predicting its possible effect on health.

In 1971, during the production of Pruteen on a semi-technical installation, an incident occurred in which several men were exposed to an aerosol of culture containing whole organisms. Altogether 14 men were affected, and they all developed influenzalike symptoms, including headaches, aching limbs, shivering, and vague tightness of the chest. These symptoms occurred during the evening and night after exposure and subsided completely over the next day. The serum of the men affected was tested 
subsequently for precipitating antibodies to Pruteen, and these were shown to be present in 11 of the 14 men (J T B Bain, personal communication).

Subsequently, several incidents have occurred in which men have been exposed to high atmospheric dust concentrations. The ensuing symptoms comprised sore, red eyes with a purulent discharge, and general symptoms similar to those of mild influenza, with headaches, aching limbs, shivering, and sometimes a feeling of tightness of the chest with slight cough. Invariably the symptoms followed exposure to high dust concentrations and occurred during the evening and night after exposure, with complete recovery within 24-48 hours. The eye symptoms occurred most frequently, the influenza-like symptoms occurring only after very heavy exposure.

Industrial hygiene and dust suppression measures have now virtually eliminated reactions during the normal production and handling of Pruteen.

\section{Materials and methods}

In 1975 a medical surveillance programme was set up to monitor the health of the work force of 106 men with particular reference to allergic response and respiratory effects.

The programme was applied to the work force already working with Pruteen and included some men who had already been working on the pilot plant for up to four years. No selection was made on medical grounds, and the population contained atopics, including those with asthma, as well as men suffering from chronic bronchitis. Attendance was voluntary but only two men refused to take part. It was not possible to include a control group, but the population was divided into three categories based on a rough estimate of their degree of exposure:

\section{Category 1:}

Continuous exposure-Plant operators who spent all their working time on the plant and who were likely to receive the highest exposures.

\section{Category 2:}

Recurrent exposure-Maintenance workers whose exposure could be heavy but intermittent.

\section{Category 3:}

Occasional exposure-Technical and supervisory staff who spent only short periods on the plant.

At the initial examination the men were asked to complete a questionnaire based on that of the Medical Research Council and including questions on occupational dust exposure, respiratory illness, smoking habits, and history of allergy. Smokers were divided into heavy smokers (over 15 cigarettes/day) and light smokers.

Skin prick tests were used to identify atopy, using standard Bencard solutions for grass and pollen, house dust, and Aspergillus fumigatus. An atopic was defined as anyone having a positive reaction to one or more of the tests, or with a history of allergy elicited by the questionnaire.

To detect sensitisation to Pruteen, an antigen extract was used which was prepared from sonicated $12.5 \%$ suspensions of Pruteen in buffer $\mathrm{pH} \mathrm{9.6}$ $(0.15 \mathrm{M})$ and $\mathrm{pH} 5(0.05 \mathrm{M})$, both being centrifuged and mixed in equal quantities.

The presence of precipitating antibodies to Pruteen was detected in the serum from venous blood samples by simple Agar diffusion methods, using extracts of the organism and the final product.

Lung function was assessed by measuring $\mathrm{FEV}_{1}$ and FVC with a Vitalograph spirometer, and the gas transfer factor was measured with a Morgan respirometer. The measurements were all carried out by one trained technician, and the equipment was calibrated annually. Because of shift working and the demands of work on the plant it was possible to examine the men in groups of only 10 to 12 at a time. Sessions were held between 0900 and 1100 on Friday each week, and the initial programme extended over several months. The men were asked not to smoke for one hour before their examination.

Finally, everyone included in the programme participated in the annual mass miniature $x$-ray screening programme on the site. At these examinations the author interviewed the men and recorded any symptoms experienced since their last interview with particular reference to eye and respiratory symptoms. They were tested for sensitisation by skin prick test, and for the presence of precipitating antibodies, and lung function tests were repeated. New employees on the plant were seen and included in the surveillance programme on starting employment.

Atmospheric dust measurements taken on the plant were recorded, and all incidents involving exposure to high dust concentrations were recorded and investigated.

\section{Results}

POPULATION

Of the 104 men initially concerned in the programme, 26 left the project in the first four years. One man died (of a myocardial infarction) while seven retired on reaching retirement age, 11 were transferred to other works on the site, and seven left the company. There is no evidence that anyone left the project for medical reasons related to exposure to Pruteen. Of 
the 78 remaining subjects, eight had incomplete data recorded; the residual population of 70 was therefore used for analysis of the results.

Over the four years, a further 81 men have worked on the project and have been added to the survey but have not been included in these results. Of the residual population of 70,20 were identified as atopics, 18 were heavy smokers and 15 light smokers, and with regard to exposure, 26 were assigned to category 1,28 to category 2 , and 16 to category 3 .

\section{SYMPTOMS}

Eleven men complained that at some time they had experienced eye symptoms after exposure. Of these, four said that they had had more than one attack and thought that they had reacted more readily than their work mates. It was noticed that when several men were exposed to high concentrations of dust on the same occasion, some did not experience symptoms.

Four men complained of having experienced influenza-like symptoms with some tightness of the chest; in three cases they also had "sticky eyes." Only one of these men was identified as an atopic, and none showed any evidence of sensitisation by skin prick test. Similarly, no one developed precipitating antibodies in association with his symptoms, either at the time of exposure or subsequently.

\section{SKIN PRICK TEST}

No one in the survey has shown a positive reaction to the skin prick test for Pruteen.

\section{PRECIPITIN TEST}

Only one man has produced a positive reaction. This man was affected in the incident in 1971 and developed antibodies at that time, which have been detectable throughout the four years of the survey. No one has developed antibodies during the survey period.

\section{RADIOGRAPHY}

The films were all examined by a consultant radiologist, and no serious abnormalities were detected.

\section{LUNG FUNCTION}

The lung function data were analysed in an attempt to detect group exposure effects that were not apparent from the individual clinical records. For the 70 men in the study, the differences between the most recent and the initial lung function readings were used to calculate annual loss rates for $F E V_{1}$ and FVC in $\mathrm{ml} /$ year and gas transfer factor $(\mathrm{TL})$ in $\mathrm{ml} / \mathrm{min}$ per $\mathrm{mm} \mathrm{Hg}$ a year. Mean values for these loss rates were then evaluated for the three categories of exposure, the three categories of smoking and the two categories of atopy after ascertaining that there were no misleading interactions among these groups. The mean annual loss rates for the 26 continuously exposed men were 47 for $\mathrm{FEV}_{1}, \mathbf{9 0}$ for FVC, and 0.25 for $T_{L}$. Taking into account the small numbers of men concerned, and the large standard errors of these mean values, they are not dissimilar from those for the 28 recurrently exposed men $(54,64$, and 0 respectively) or the 16 occasionally exposed men $(33,75$, and $0 \cdot 24$ respectively). By the same criteria, the differences between the mean values for the 20 atopics $(52,82$, and $0 \cdot 14$ respectively) are barely distinguishable from those for the other 50 men (44, 74, and $0 \cdot 14$ respectively). Analysis for the three smoking groups also yielded similar figures. The means for the 18 heavy smokers were 51,102 , and $0 \cdot 06$; for the 15 light smokers 55,81 , and $0 \cdot 17$; and for the 37 non-smokers 42,62 , and $0 \cdot 19$.

\section{Discussion}

As a result of the medical surveillance programme, it has been possible to identify and evaluate the effects of exposure to high concentrations of Pruteen dust. The symptoms experienced fall into two distinct types: a conjunctivitis with profuse sterile purulent discharge, and general febrile symptoms resembling a mild attack of influenza. Invariably they occur several hours after exposure, and complete recovery takes place within 24 to 48 hours. Some men appear to experience these symptoms more readily than others.

Symptoms occur only after exposure to high concentrations of dust and have not been reported during normal working conditions. When atmospheric dust has been measured the lowest recorded concentration at which symptoms have been produced has been $10 \mathrm{mg} / \mathrm{m}^{3}$. The nature of the response is not fully understood. Tests for allergy suggest that the dust is non-allergenic. Skin prick tests have repeatedly shown the absence of sensitisation, there have been no symptoms suggestive of a type I allergy, and atopics have not proved more susceptible than non-atopics. Tests for precipitating antibodies to Pruteen have also been repeatedly negative, and the symptoms do not suggest a type III allergic reaction.

In the incident in 1971 men were exposed to an aerosol of whole organisms, and antibodies were present in most of the men. Immunisation studies in animals have shown that Pruteen contains far fewer antigens than does the parent organism; it seems that the immunogenicity of the organism is considerably reduced in the subsequent processing of the culture.

The lung function measurements are of value for assessing subclinical effects. Berry ${ }^{3}$ has shown that large numbers of men must be monitored over long 
periods if anything but gross effects are to be shown to be statistically significant, while Fletcher et $a l^{4}$ have discussed a wide range of factors that can cause such analyses to be misleading. Nevertheless, had a pronounced dose-effect relationship been observed for these 70 men over the period of four years it could have been regarded as the first indication of a chronic pulmonary problem.

In practice, the differences observed among the various exposure, smoking, and atopic categories are so small, by comparison with the variability in the data, as to make any interpretation extremely premature.

The overall mean rate of loss of $\mathrm{FEV}_{1}$ for the 70 men, $46 \mathrm{ml} /$ year, is in line with published figures for studies inclusive of smokers, and the mean loss rate for gas transfer factor is close to its predicted value. The mean rate of loss of FVC, $76 \mathrm{ml} /$ year, is high but, if it is correlated with anything, it is with smoking habits rather than with exposure category or atopic status.

Probably symptoms are produced as a reaction to exposure to the lipopolysaccharide (endotoxin). It has been shown experimentally in animals that Pruteen is capable of activating complement by the alternative pathway, but attempts to show $\mathrm{C}_{3}$ conversion in exposed men have so far been unsuccessful. Attempts to reproduce the eye symptoms in rabbits or guinea-pigs by exposure to direct contact with the dust have also been unsuccessful (G E Davies, personal communication).
The results have shown that Pruteen can be produced and handled without adverse effects on health, provided that adequate measures are taken to prevent exposure to high concentrations of dust.

I thank Dr G E Davies, ICI Central Toxicology Laboratory, who carried out the immunological work, and Dr G M Paddle, medical statistician, ICI Central Medical Group, who did the statistical analyses, for their invaluable advice and support. I also thank Dr P T Monard, medical officer, ICI, for his help in designing the programme and Dr C A C Pickering, Wythenshawe Hospital, for his guidance and help.

Finally, I thank the staff of the Billingham medical department and the men who participated in the survey for their willing co-operation and interest.

\section{References}

1 Parkes WR. Occupational lung disorders. London: Butterworths, 1975:392.

2 Juniper CP, How MJ, Goodwin BFJ, Kinshott AK. Bacillus subtilis enzymes: a 7-year clinical, epidemiological and immunological study of an industrial allergen J Soc Occup Med 1977;27:3-12.

${ }^{3}$ Berry G. Longitudinal observations, their usefulness and limitations with special reference to the forced expiratory volume. Bull Physio-path Resp 1974;10:643-55.

${ }^{4}$ Fletcher $\mathrm{C}$, Peto $\mathrm{R}$, Tinker C, Speizer FE. The natural history of chronic bronchitis and emphysema. Oxford: Oxford University Press, 1976.

\section{CORRECTIONS}

\section{Healthy worker effect in the total Finnish population (May 1980)}

On page 181 the last paragraph should read: "Of the 4028 who stayed within the same occupational group, 151 died" as is shown in table 4.

\section{A study of the mortality of Cornish tin miners (November 1981)}

The recommended standard for men exposed to radon in underground air should have read four working level months (p. 380). The conclusions of the paper are unaffected by this error.
Relationship between type of simple coalworkers' pneumoconiosis and lung function. A nine-year followup study of subjects with small rounded opacities (November 1981)

The authors regret that in their tables 4-7 the units of compliance and recoil pressure while reported to be in SI units were in fact given in traditional units; to make the conversion the numerical values for compliance and recoil pressure should respectively be multiplied by and divided by 10 . 